\title{
Estudio comparativo técnico y económico del sistema de perforación horizontal ramming y el sistema convencional, estudio de caso
}

\section{Comparative Technical and Economic Study between Pipe Ramming and the Conventional System: Case Study}

\author{
Saieth Baudilio Chaves-Pabón ${ }^{1} \bowtie$, Dayan Cárdenas-Moreno², \\ Carolina Avilez-Romero ${ }^{3}$, Julieth Barajas-Bernal ${ }^{4}$
}

\author{
${ }^{1}$ Universidad Militar Nueva Granada, Bogotá, Colombia \\ 2 Universidad Católica de Colombia, Bogotá, Colombia \\ ${ }^{3}$ Universidad Católica de Colombia, Bogotá, Colombia \\ ${ }^{4}$ Universidad Católica de Colombia, Bogotá, Colombia
}

$\square$ Facultad de Estudios a Distancia, Universidad Militar Nueva Granada, Programa de Ingeniería Civil. Kilómetro 2 de la vía Cajicá-Zipaquirá, Cajicá, Cundinamarca, Colombia. Email: saieth.chaves@unimilitar.edu.co

Recibido: 5 de septiembre del 2017 Aprobado: 1 de diciembre del 2017 Disponible en línea: 1 de enero del 2018

How to cite this article: S. B. Chaves-Pabón, D. Cárdenas-Moreno, C. Avilez-Romero y J. Barajas-Bernal, "Estudio comparativo técnico y económico del sistema de perforación horizontal ramming y el sistema convencional, estudio de caso", Revista Ingeniería Solidaria, vol. 14, no. 24, pp. 16, enero 2018. doi: https://doi.org/10.16925/in.v14i24.2165

\section{Resumen}

Introducción: el artículo es el resultado de la investigación "Estudio comparativo técnico y económico del sistema de perforación horizontal "ramming" y el sistema convencional, estudio de caso", desarrollada en la Universidad Católica de Colombia durante el 2016.

Objetivo: realizar una comparación técnica y económica al tomar como referencia una obra ubicada en el municipio de Tocancipá, mediante la cual se identifiquen cantidades de obra, costos, procesos constructivos, impacto ambiental, equipos y mano de obra en obras de instalación de redes subterráneas.

Metodología: consistió en recopilación de información bibliográfica sobre el tema, adquisición y análisis de datos de campo y presentar conclusiones ordenadas y claras con el fin de cumplir el propósito principal del proyecto.

Resultados: a partir de los datos de campo se obtuvieron otros que permitieron realizar la comparación económica de los costos directos e indirectos; de igual forma, en los aspectos técnicos de ambas tecnologías de intervención.

Conclusiones: se logró hallar los costos directos e indirectos en los que se evidencia cómo para los costos directos el sistema de excavación a cielo abierto es más económico que el sistema de excavación ramming, y para los costos indirectos sucedió lo contrario.

Originalidad: el proyecto tiene un aporte de originalidad y de interés en la ingeniería civil dado que fue posible realizar un estudio completo en una obra con dos técnicas de cruce de tuberías y se ha entregado un insumo importante para desarrollar futuras investigaciones.

Limitaciones: escaza información sobre la utilización e investigación publicada de nuevas tecnologías de construcción de cruces de tuberías en Colombia.

Palabras clave: estudio comparativo, excavación en zanja, ramming, redes subterráneas, tecnología. 


\title{
Comparative Technical and Economic Study between Pipe Ramming and the Conventional System: Case Study
}

\begin{abstract}
Introduction: The article is the result of the investigation "Technical and economic comparative study of the system of horizontal perforation "ramming" and the conventional system, case study", developed in the Universidad Católica de Colombia during the year 2016.

Objective: Perform a technical and economic comparison with reference to a construction site located in the municipality of Tocancipá, which identify amounts of work, costs, construction processes, environmental impact, equipment, and construction workers, of the systems referred to in construction sites of underground networks.

Methodology: It consisted of a collection of bibliographic information on the theme, acquisition and analysis of field data, and present conclusions clear and ordered fulfilling with the main purpose of the project.

Results: The information of field data were obtained to make the economic comparison of direct and indirect costs, also failed to make a parallelism in the technical aspects of both technologies of intervention and finally they determined the execution times.

Conclusion: Managed to find the direct and indirect costs where there is evidence that for direct costs open pit system is more economical than the excavation system, and for the indirect costs it happened otherwise. The project is a contribution of originality and interest in civil engineering since it has managed to do a complete study on a work with two techniques of intersection of pipes and an important input for developing future research has delivered. The limitation is the scarce information about the use and published research of new technologies of construction of crossings of pipelines in Colombia.
\end{abstract}

Keywords: Comparative study, ditch digging, ramming, underground networks, technology.

\section{Estudo comparativo técnico e econômico do sistema de perfuração horizontal ramming e o sistema convencional: estudo de caso}

\section{Resumo}

Introdução: este artigo é resultado da pesquisa "Estudo comparativo técnico e econômico do sistema de perfuração horizontal 'ramming' e o sistema convencional, estudo de caso", desenvolvida na Universidad Católica de Colombia, em 2016.

Objetivo: realizar uma comparação técnica e econômica ao tomar como referência uma obra localizada no município de Tocancipá (Colômbia), mediante a qual são identificadas quantidades de obra, custos, processos construtivos, impacto ambiental, equipes e mão de obra em obras de instalação de redes subterrâneas.

Metodologia: consistiu em recopilação de informação bibliográfica sobre o tema, aquisição e análise de dados de campo e apresentação de conclusões ordenadas e claras a fim de cumprir o objetivo principal do projeto.

Resultados: a partir dos dados de campo, foram obtidos outros que permitiram realizar a comparação econômica dos custos diretos e indiretos; de igual forma, foram obtidos os aspectos técnicos de ambas as tecnologias de intervenção.

Conclusões: pôde-se encontrar os custos diretos e indiretos nos quais se evidencia como, para os custos diretos, o sistema de escavação a céu aberto é mais econômico que o sistema de escavação ramming; para os custos indiretos, aconteceu o contrário.

Originalidade: 0 projeto tem uma contribuição de originalidade e de interesse na engenharia civil por ter sido possivel realizar um estudo completo numa obra com duas técnicas de cruzamento de tubulações e entregou-se um insumo importante para desenvolver futuras pesquisas.

Limitações: há escassa informação sobre a utilização de novas tecnologias de construção de cruzamento de tubulações na Colômbia e pesquisa publicada a respeito do tema.

Palavras-chave: estudo comparativo, escavação em vala, ramming, redes subterrâneas, tecnologia. 


\section{Introducción}

El desarrollo y crecimiento de las ciudades ha impulsado la demanda de ejecución de nuevas redes públicas y privadas subterráneas, principalmente, en zonas con alta densidad de redes existentes. De esta manera, se ha incrementado la necesidad de crear nuevos sistemas que puedan ir de forma subterránea sin alterar las actuales líneas de servicios.

De acuerdo con lo anterior, en el presente trabajo de investigación se pretendió hacer una comparación técnica y económica [1, p. 19] entre el sistema convencional usando zanjas o perforación a cielo abierto [2, p. 14], [3, p. 39], y el uso de tecnologías sin zanjas por medio del sistema ramming [4, p. $1 ; 5$, p. 5], tomando como referencia el caso de "La construcción de obras civiles para la expansión y mejoramiento del servicio de energía eléctrica del municipio de Tocancipá, Colombia”, bajo el Contrato n. ${ }^{\circ} 196$ de 2015, en el cual se implementaron los dos sistemas.

A partir del análisis del caso de estudio, la recopilación de información suministrada por la empresa encargada de le ejecución del proyecto en lo referente a los aspectos técnicos y económicos, se determinaron cantidades de obra, tiempo de ejecución y costos de las actividades que componen cada uno de los métodos en estudio, con el fin de realizar un análisis del impacto generado teniendo en cuenta que el sitio donde se desarrolló este proyecto es una zona industrial.

Cabe considerar las afectaciones que se pueden producir en una ciudad cuando se realizan obras viales como, por ejemplo, cortes en la circulación, accidentes e inseguridad, lo cual ha generado un interés especial en la necesidad de implementar planes que aporten al mejoramiento sostenible de la calidad de vida de la población y, como propósito, mejorar la competitividad de la industria de la construcción, incluyendo obras subterráneas de redes de saneamiento para el sector del área urbana.

Para la subterranización de la red eléctrica del proyecto en estudio, fue necesario realizar perforación por el sistema ramming, debido a que el área intervenida es una zona industrial donde se encuentran ubicadas varias empresas y también vías principales. Al no hacer estas perforaciones subterráneas se generaba un gran impacto negativo en todas las industrias puesto que se producirían cierres de vías, lo que afectaba directamente a las empresas en asuntos tales como retrasos en cadena de producción y de logística, y de forma indirecta a todas las personas que requirieran transitar por esta zona. A su vez, en los demás tramos del proyecto se realizaron actividades relacionadas con el cruzamiento de la red eléctrica por medio del sistema convencional de excavación a cielo abierto.

Dada esta situación, surgió la oportunidad y la necesidad de realizar un comparativo entre estos dos sistemas [6, p. 2] y así determinar los impactos producidos por cada uno de los sistemas utilizados en relación con los aspectos técnicos y económicos, estudio que no se había realizado hasta el momento y que permitió identificar los pro y los contra del uso de cada uno de estos métodos de trabajo para el tipo de obras en estudio. En consecuencia, se realizó una comparación técnica y económica tomando como referencia el caso de estudio de la obra ubicada en el municipio de Tocancipá, Cundinamarca (Colombia), mediante el cual se identificaron: cantidades de obra, costos, procesos constructivos, impacto ambiental, equipos y mano de obra tanto del uso del sistema de perforación horizontal ramming como del sistema convencional a cielo abierto en obras de instalación de redes subterráneas $[7, \mathrm{p}$. 28], [8, p. 11]. Las fases previstas para el proyecto consistieron en la identificación y documentación de toda la información pertinente (usos, procedimiento, beneficios, ventajas, etc.) de las tecnologías sin zanjas mediante el sistema ramming; en la segunda fase se efectuó un análisis del impacto que causan las instalaciones de redes subterráneas por medio del sistema tradicional de excavaciones a cielo abierto y el aporte que trae la implementación de un sistema de perforación horizontal ramming [9, p. 12], [10, p. 30]. Finalmente, se realizó un comparativo de los aspectos técnicos y económicos al realizar la construcción de obras de redes subterráneas, con excavaciones a cielo abierto y con el uso del sistema ramming, así como los impactos que cada uno de estos puede llegar a generar.

\subsection{Estado del arte}

En relación con la definición del método pipe ramming diversos autores han expuesto sus propuestas. De acuerdo con Abraham, Baik y Gokhale [11, p. 9], pipe ramming es un método no destructivo para la instalación de tubería y revestimientos de acero, en el cual un martillo neumático se utiliza 
a fin de hincar la tubería o los revestimientos en el suelo. Según Howell [12, p. 255] esta tecnología básicamente consiste en hincar una tubería de acero e impulsarla gracias a los impactos sucesivos proporcionados por una máquina de percusión, como, por ejemplo, un martillo neumático. Collins $[13$, p. 2] manifiesta que este método se utiliza para instalar las tuberías de forma horizontal, pero en algunas ocasiones se pueden situar verticalmente $o$ con inclinación. Por su parte, Stuedling y Tadesse $[14$, p. 126] en sus investigaciones concluyen que la instalación de nuevas tuberías destinadas a redes de alcantarillados, redes de agua potable, redes de conducción de gas o redes de conducción eléctrica, entre otras, así como la sustitución de las redes existentes empleando nuevas tecnologías sin zanjas, entre ellas, la tecnología ramming, aumentan en notoriedad frente a los métodos convencionales como el sistema tradicional de excavación a cielo abierto. Todo lo anterior debido a que es una técnica eficiente y de bajo impacto en las actividades cotidianas que se puedan desarrollar en el entorno de la zona de trabajo. En otras publicaciones científicas autores como Milligan y Rogers [15, p. 570], Kramer, Thomson y McDonald [16, p. 130], y Stein [17, p. 51], han profundizado en esta técnica y logran demostrar que el alcance de estas nuevas tecnologías ha ido variando desde una técnica simple de percusión hasta altos procesos sofisticados que involucran excavaciones balanceadas o perforación dirigida mediante equipos robotizados. Si bien estas técnicas no serán descritas en este artículo, se pueden consultar para futuros propuestas de investigación en nuestro país.

En relación con la comparación de los métodos de cruzamiento de tuberías o conducciones, convencional y pipe ramming, diversos autores han manifestado las bondades del método pipe ramming, principalmente por los beneficios a los usuarios. Según Ariaratnam, Chan y Choi [18, p. 136] en la instalación de nuevos alcantarillados o la sustitución de existentes tradicionalmente se utilizan técnicas de construcción de zanja abierta; sin embargo, es hoy muy impopular debido al impacto negativo en la vida diaria de los usuarios de carreteras ya que genera retrasos en el tráfico, desvíos, cierres de carreteras o ferrocarriles, ruidos indeseables, pérdida del acceso a las viviendas o a los lugares de trabajo, así como a la duración en la excavación, instalación y operaciones de rellenos de la zanja. Del mismo modo, Meskele y Stuedlein
[19, p. 2] destacaron que el método pipe ramming es una técnica de construcción sin zanjas eficiente y rentable utilizada, primordialmente, en aplicaciones horizontales o casi horizontales en las que la tubería es presionada al interior del terreno con unos golpes percusivos de alta frecuencia generados por un potente martillo neumático o hidráulico. Stuedlein y Meskele [14, p. 133] en otra publicación señalan que el pipe ramming permite la instalación de tuberías en suelos con partículas de gran tamaño, lo cual podría ser una condición del suelo que limitaría el uso de otras técnicas de excavación sin zanjas. Entre las diferentes tecnologías sin zanjas disponibles, tales como micro-túneles y perforación horizontal dirigida (HDD), el pipe ramming es el método preferido para la instalación de tuberías o alcantarillados bajo carreteras y vías férreas, dado que otros métodos generan gran cantidad de movimiento de tierras que podrían ser inadecuadas. De otra parte, Najafi [20, p.5] afirma que quizá el beneficio más significativo de esta técnica se encuentra en el costo-beneficio de la operación, relacionado con otras tecnologías sin zanjas, ya que requiere un equipamiento y diseño sencillo, por ejemplo, menos utilización de cabezales de rotación de corte y actuadores de elevación. Otro proyecto de investigación interesante fue llevado a cabo por Matthews, Allouche y Sterling [21, p. 196], quienes al comparar dos proyectos de construcción de cruces de tuberías con zanja abierta y sin zanjas encontraron que la inclusión de los costos sociales en la estimación del costo del proyecto podría situar la tecnología sin zanjas como más ventajosa en comparación con la construcción a cielo abierto; esto fue más significativo para áreas urbanas altamente densas respecto a áreas medianamente densas. Otro aspecto que ha resultado importante resaltar es que en áreas urbanas densas encontraron que los costos de demora por viaje es el factor más importante de los costos sociales (55\%). Jung y Sinha [ 22 , p. 145] señalan que para determinar los costos totales de un proyecto de cruce de tuberías, bien fuese por el método convencional a cielo abierto o bien sin zanjas, se deberían tener en cuenta los costos totales, los costos sociales, los costos ambientales y otros costos. $\mathrm{Al}$ ser los costos directos aquellos que implican la ejecución de la obra, los costos sociales relacionados con los retrasos del tráfico, pérdidas de los negocios y de los usuarios de la vía, los costos ambientales tales como la contaminación por ruido, aire, agua y demás, y los otros costos podrían ser la 
productividad, la seguridad y el comportamiento estructural.

Otro aspecto que se investiga en los últimos años - y no menos importante- es el efecto ambiental que tiene cada uno de los métodos de construcción. Según estudios hechos por Gerasimova [23, p. 1396], a diferencia de los métodos tradicionales de construcción de zanjas abiertas que aumentan considerablemente la contaminación del medio ambiente, los métodos de construcción sin zanjas reducen significativamente las emisiones de $\mathrm{CO} 2 \mathrm{y}$ otros gases nocivos a la atmosfera que causan enormes problemas de salud; asimismo, la contaminación por ruido es otro problema asociado a esta tecnología. De acuerdo con Ariaratnam [24, p. 10] la construcción sin zanjas tiene una ejecución más rápida y más económica, ya que ejecutar un cruce de tubería sin zanjas puede tener una duración de aproximadamente tres días, y para el método de cielo abierto puede durar aproximadamente siete. En este sentido, la North American Society for Trenchless Technology (NASTT) $[25$, p. 1] señala en su investigación cuantitativa de las ventajas ecológicas de las tecnologías sin zanjas que estas provee una reducción del $90 \%$ en la emisión de gases de efecto invernadero, y hasta un $25-30 \%$ en ahorro de los costos, comparadas con la tecnología de cielo abierto. Otros investigadores como Myers, Stickrod, Abraham e Iseley [26, p. 62] han definido los impactos sociales y ambientales del método convencional de construcción a cielo abierto: el inconveniente vehicular, el tráfico de peatones, la seguridad en el trabajo, la interrupción de los comercios, el impacto en los residentes, el impacto en la autopistas, el impacto en el subsuelo, la disposición del material de excavación, la contaminación del aire, la contaminación del agua, la contaminación por ruido y los daños en el paisaje.

\section{Materiales y métodos}

\subsection{Excavación a cielo abierto o convencional}

Teóricamente, es aquella que se ejecuta en condiciones naturales de iluminación, ventilación y drenaje. Las excavaciones consisten en la extracción de materiales en sitios previamente definidos y en los que la zanja debe ser lo suficientemente ancha para permitir al personal trabajar en condiciones de seguridad [27, p. 34]. En el caso de estudio, el ancho mínimo (Bd, según se aprecia en la figura 1) ha sido el diámetro exterior más $0,3 \mathrm{~m}$ y, para el promedio, el diámetro exterior más $0,40 \mathrm{~m}$. Si se hubiese requerido ampliar el ancho de la zanja debe hacerse por encima del lomo de la tubería.

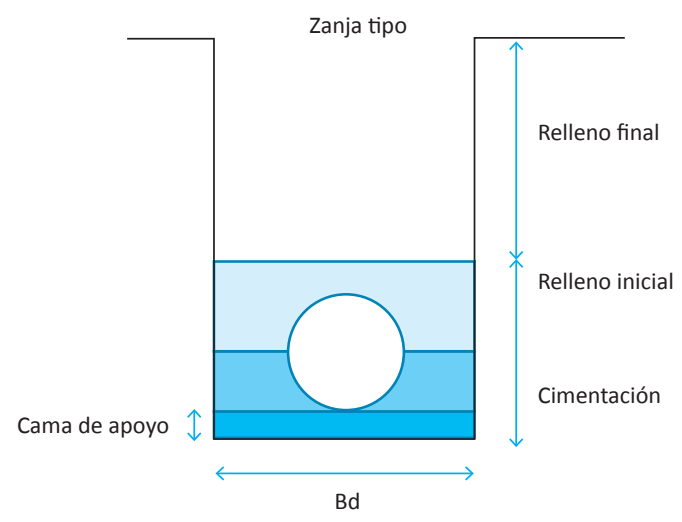

Figura 1. Excavación tipo en zanja o convencional Fuente: elaboración propia

\subsection{Excavación por el sistema ramming}

También conocido como pipe ramming es una técnica de instalación de tuberías sin zanjas utilizada para hincar horizontalmente tubería de hacer de diferentes diámetros. La principal diferencia con respecto a la hinca de tubería "normal" es que el empuje se realiza mediante un martillo neumático o hidráulico que golpea el tubo de hacer, el cual penetra en suelo sin alterarlo [28, p. 31].

La palabra ramming viene del gerundio del verbo ram que traduce "golpear duro" o "apisonar", el cual, aplicado al sistema relacionado en el presente estudio se ve directamente relacionado con su proceso constructivo. El avance de esta tecnología en el sector de la ingeniería y su aplicación se debe, principalmente, al impacto que genera este tipo de obras en la sociedad en aspectos tales como: las profundas consecuencias negativas desde el punto de vista ambiental, social y económico generadas por las excavaciones a cielo abierto; el excavar sobre una calzada, los ruidos que producen los martillos neumáticos por la rotura del pavimento, los cambios de tipo y colores en los parcheos sobre el pavimento; el hecho de que una obra por pequeña que sea se demora más de lo previsto en su ejecución; la remoción de los volúmenes de material de excavación y 
relleno que generan gran cantidad de polvo, olores y gases; el aumento en la interrupción del tráfico vehicular $y$, en muchas ocasiones, peatonal, lo que genera un gran malestar en la comunidad residente y aledaña al lugar donde se está realizando la obra; $y$, peor aún, cuando se trata de una zona comercial o industrial en la que por razón de estos inconvenientes afecta la actividad económica de estos establecimientos aledaños [29, p. 43].

A fin de minimizar los impactos anteriores se requiere documentar con la información pertinente los usos, el proceso constructivo, las características técnicas, las limitaciones, las condiciones requeridas y las ventajas del sistema. El funcionamiento del sistema ramming es de empuje dinámico mediante golpes, los cuales hacen que la camisa de acero hinque de manera progresiva y se logre completar la longitud requerida para llevar a cabo el cruce.

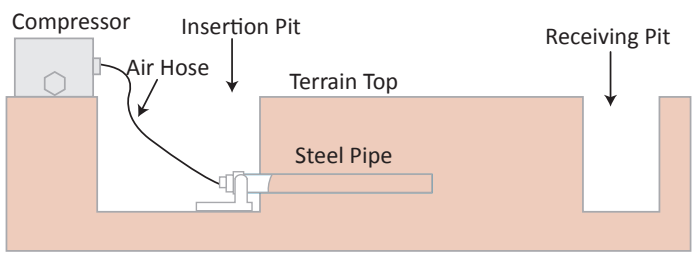

Figura 2. Esquema general de instalación de tuberías por medio del sistema ramming

Fuente: [30]

Se conocen instalaciones hasta de $3000 \mathrm{~mm}$ de diámetro en terrenos con condiciones óptimas realizadas con martillos de hinca de $800 \mathrm{~mm}$ de diámetro que llegan a generar fuerzas de hasta 40500 Nm. Sin embargo, como es común en las tecnologías sin zanja esto puede variar pues depende mucho de las condiciones del terreno y de la experiencia del operador de la maquinaria. Cuando se utiliza HDD es común que en el proceso se encuentren variaciones del terreno, variaciones que ni siquiera se contemplaban en los estudios geológicos, o casos como cambios en niveles freáticos y aparición de nuevos tipos de terreno [31, p. 35].

En el caso base del proyecto "Expansión y mejoramiento del servicio de energía eléctrica", en el municipio de Tocancipá, se requirieron seis tramos con el sistema ramming, así: tramo n. ${ }^{\circ} 1$, entrada Parque Industrial Gran Sabana, diámetro de 24 " y longitud de 51,15 m; Tramo n. ${ }^{\circ} 2$, frente al Parque Industrial Gran Sabana, diámetro de 24" y longitud de $40 \mathrm{~m}$; Tramo n. ${ }^{\circ}$ 3, frente al Parque Industrial Gran Sabana, diámetro de 24" y longitud de 18,50 m; Tramo n. ${ }^{\circ} 4$, salida Parque Industrial Gran Sabana, diámetro de 30" y longitud de $16 \mathrm{~m}$; Tramo n. ${ }^{\circ}$, Cruce vía férrea, diámetro de 36 y longitud de $42 \mathrm{~m}$; Tramo n. ${ }^{\circ}$ 6, frente a Foster, diámetro de 24 " y longitud de $42,50 \mathrm{~m}$, en este tramo se convino con el sistema de Túnel Linner por limitaciones de espacio para el pozo. Los tramos anteriores se realizaron con este sistema ramming debido a sus implicaciones de ubicación, lo cual afectaba la calzada de acceso y salida vehicular y peatonal de predios industriales como el Parque Industrial Gran Sabana, Foster, Ebel, etc.

\section{3 Área de estudio}

Para el caso de estudio se tomó como referencia el contrato 196 de 2015, celebrado en el municipio de Tocancipá, situado en el Parque Industrial Gran Sabana, en el cual se realizaron seis cruces de vías en perforación por el sistema ramming para la subterranización de la red eléctrica; se empleó una camisa en acero de 24 " para 6Ø6" y 30 " para 9Ø6”, y 36" para 12 Ø6” de diámetro, y cada cruce tuvo una longitud aproximada entre 30 y $40 \mathrm{~m}$ que corresponden al ancho de las vías. Al realizar la instalación de la red eléctrica en estos cruces de vías a cielo abierto se evitó generar cierres de vías que pudieran causar molestias a las industrias aledañas al sector intervenido. Asimismo, el desarrollo de los demás tramos intervenidos en el proyecto, debido a la zona de ubicación, se pudieron llevar a cabo mediante excavación a cielo abierto, razón por la cual se tomó como referencia este proyecto para el tema de estudio de la presente investigación.

\subsection{Metodología}

La presente investigación tuvo un enfoque cualitativo dado que la fuente de información será recolectada del proyecto de construcción de obras civiles de expansión y mejoramiento del servicio de energía eléctrica en el municipio de Tocancipá. Se empleó el sistema de perforación horizontal ramming en seis tramos del recorrido, y el sistema convencional excavación a cielo abierto en los demás tramos que hacen parte del proyecto. Con 
base en la información recopilada se realizó una comparación técnica y económica que determinó cantidades de obra, tiempo de ejecución, costos, impacto ambiental, equipo, mano de obra y procesos constructivos de las actividades que componen cada uno de los métodos en estudio, desarrollado mediante tres fases: recopilación de información, seguimiento caso de estudio y, finalmente, la elaboración de la comparación.

En la primera fase del proyecto se recopiló la información, los antecedentes y el impacto que se produjo en proyectos que se habían realizado con el uso de sistema ramming, específicamente el caso en estudio para el municipio de Tocancipá, y de la empresa Ingeniería Redes y Túneles sas, dedicada a la instalación de redes subterráneas. Asimismo, se logró el apoyo y la colaboración con los profesionales de la empresa en lo referente a la ejecución de estos procesos y la bibliografía existente con relación al tema.

Durante la segunda fase del trabajo de investigación se realizó el seguimiento al caso en estudio, y se determinaron aspectos tales como: procedimiento, ventajas, desventajas e impacto ambiental, social y económico tanto en la aplicación del sistema de excavaciones a cielo abierto como en la aplicación del sistema ramming, lo que generó en la construcción de obras civiles expansión y mejoramiento del servicio de energía eléctrica del municipio de Tocancipá.

En la fase final del proyecto, durante la cual se consolidó la información recopilada y se elaboró la comparación técnica y económica con base en el caso de la obra ubicada en Tocancipá, se determinó cantidades de obra, tiempo de ejecución, costos, impacto ambiental, equipo, mano de obra y procesos constructivos de las actividades que componen cada uno de los métodos en estudio. Con el fin realizar un análisis del impacto que se genera teniendo en cuenta que el sitio donde se desarrolló este proyecto es una zona industrial.

Ahora bien, en el análisis económico de este tipo de obras subterráneas cabe cuestionar la responsabilidad que acarrean los costos de los prejuicios colaterales, hecho que influyó en el contexto directo de afectación del proyecto. Estos, normalmente, no se asumen por parte del contratista debido a que este asume los costos directos de la obra y los indirectos de la administración que requiere el cumplimiento del alcance de un contrato de obra. Según lo anterior estos costos son normalmente asumidos por los directamente afectados y sus futuras generaciones.

Esta situación genera un concepto erróneo que beneficia por un costo menor a la opción tradicional a cielo abierto, cuando de hecho es comprobable que al costear los costos ambientales y sociales que ocasiona este tipo de obras se pueden llegar a percibir costos mayores a la implementación de una tecnología más avanzada como el sistema ramming.

Cuando se realiza una comparación entre un sistema tradicional y un sistema de perforación horizontal como el ramming, este último ofrece beneficios ambientales, sociales y financieros en conformidad con un avance sustentable en el desarrollo urbano de una ciudad.

Otro punto clave en este análisis es el tiempo, debido a que una obra a cielo abierto requiere mayor tiempo, lo cual crea directamente una frustración para los ciudadanos que circulan o habitan el área aferente de la obra. Debido al tiempo requerido, es indispensable disponer de personal que implica un pago mayor en nóminas, seguridad social y dotación; igualmente, habría que contabilizar el tiempo de alquiler de equipos usados.

$\mathrm{Al}$ analizar el campo de seguridad y salud de trabajo (ssт) del personal, cabe considerar que en obras subterráneas realizadas por el sistema tradicional el riesgo de accidentabilidad es mayor debido a que se requiere trabajar en espacios confinados. Además, se debe tener cuenta el riesgo que se puede producir al trabajar cerca de la circulación de tráfico vehicular. Mientras que para la implementación del sistema de ramming la excavación es menor, es confinada y se aparta al personal de trabajar sobre la superficie, de modo que se ubica por fuera de áreas de riesgo y tráfico.

Dado lo expuesto anteriormente, a continuación, se determinan los tipos de costos que se deben tener en cuenta en este análisis, los cuales se pueden clasificar en dos tipos: costos directos - los relacionados con las actividades necesarias para la ejecución de cada uno de los procesos constructivos- y los costos indirectos - relacionados con los costos correspondientes a los prejuicios colaterales que acarrea cada método-. 


\section{Resultados}

\subsection{Comparación desde el aspecto económico}

\subsubsection{Comparación de los costos directos}

Para la realización del estudio el punto de partida fue el análisis de los costos relacionados con las actividades más relevantes dentro de la obra a cielo abierto y así estar en capacidad de compararlas con las actividades similares en el sistema ramming. Estas actividades se analizarán a continuación.

\subsubsection{Costos directos en actividades preliminares}

Al comparar las actividades preliminares requeridas para cada uno de los casos es necesario tener en cuenta la cantidad que se requiere para evidenciar una base comparativa en iguales condiciones.

Como se observa en la tabla 1 y la figura 3 se evidenció que los costos preliminares del sistema tradicional requieren mayores cantidades en las actividades de demolición, de modo que la excavación llega a ser equidistante y en el sistema con zanja no se contempla el ítem de entibados. A pesar de esto el sistema ramming tiene un mayor costo por los entibados que se requieren para las cajas de lance y recibo ya que por su profundidad es necesario confinar el área de trabajo como medida de protección del personal. Sin esta actividad se pudo observar que el sistema a cielo abierto es más costoso, pues los costos de demolición y excavación del ramming representan solo un $48 \%$ del costo del sistema tradicional.

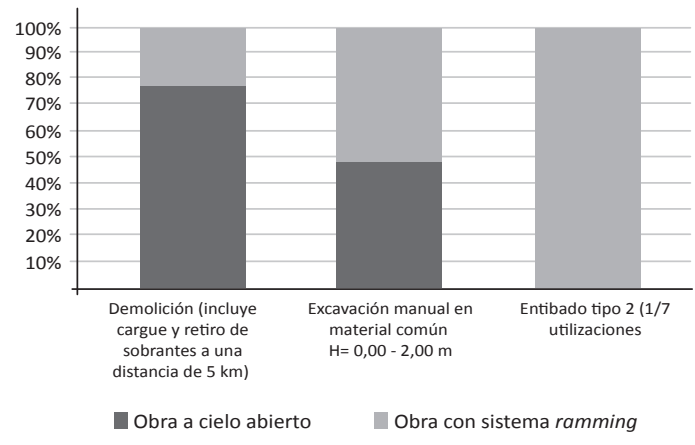

Figura 3. Comparasión de costos directos en actividades preliminares

Fuente: elaboración propia

\subsubsection{Costos directos en la instalación y suministro de tubería}

Otra actividad determinante es el suministro e instalación del colector o ducto por el cual se ha canalizado la red subterránea. En ambos sistemas se requiere la instalación de un conjunto de tubería PVC TDP según diseños. La diferencia radica en que con el sistema ramming se encamisa esta ductería por dentro de una tubería en acero al carbón que permite tener un conducto protegido y limpio que lo separa del terreno como tal.

Como se evidenció en tabla 2 y la figura 4 , en el caso en referencia se observó una diferencia de costo entre los dos tipos de sistemas. En la perforación horizontal ramming, por su tecnología y el encamisado de los ductos, se incrementa el costo representativo en un $84 \%$ más con relación al sistema tradicional a cielo abierto .

Tabla 1. Comparativo de costos actividades preliminares

\begin{tabular}{|c|c|c|c|c|c|c|}
\hline \multirow[b]{2}{*}{ Actividad } & \multicolumn{3}{|c|}{ Obra a cielo abierto } & \multicolumn{3}{|c|}{ Obra con sistema ramming } \\
\hline & Cant. & Vlr. unitario & Vlr. total & Cant. & Vlr. unitario & Vlr. total \\
\hline \multirow{2}{*}{$\begin{array}{l}\text { Demolición (incluye } \\
\text { cargue y retiro de } \\
\text { sobrantes a una } \\
\text { distancia de } 5 \mathrm{~km} \text { ) }\end{array}$} & \multicolumn{3}{|c|}{ Demolición de anden y calzada } & \multicolumn{3}{|c|}{$\begin{array}{c}\text { Demolición de anden para localizar } \\
\text { pozo de arranque y finalización }\end{array}$} \\
\hline & 180,5 & COP 45500,65 & COP 8211957,31 & 60,0 & COP 38083,79 & COP 2285027,40 \\
\hline \multirow{2}{*}{$\begin{array}{l}\text { Excavación manual } \\
\text { en material común } \\
h=0,00-2,00 \mathrm{~m}\end{array}$} & \multicolumn{3}{|c|}{$\begin{array}{c}\text { Excavación de toda la longitud } \\
\text { por un ancho de } 1,80 \mathrm{~m}\end{array}$} & \multicolumn{3}{|c|}{$\begin{array}{l}\text { Excavación de pozo de arranque } \\
\text { y finalización }\end{array}$} \\
\hline & 292,7 & COP 10382,10 & COP 3038383,86 & 300,0 & COP 10382,10 & COP 3114630,00 \\
\hline $\begin{array}{l}\text { Entibado tipo } 2 \text { (1/7 } \\
\text { utilizaciones) }\end{array}$ & 0,0 & COP 23081,33 & $\mathrm{COP}-$ & 540,0 & COP 23081,33 & COP 12463918,20 \\
\hline Total: & \multicolumn{3}{|r|}{ COP 11250341,17} & \multicolumn{3}{|r|}{ COP 17863575,60} \\
\hline
\end{tabular}


Tabla 2. Comparativo de costos en la instalación y suministro de tubería

\begin{tabular}{|c|c|c|c|c|c|c|}
\hline & \multicolumn{3}{|c|}{ Obra a cielo abierto } & \multicolumn{3}{|c|}{ Obra con sistema ramming } \\
\hline Actividad & Cant. & Vlr. unitario & Actividad & Cant. & Vlr. unitario & Actividad \\
\hline \multirow{2}{*}{$\begin{array}{l}\text { Instalación de } \\
\text { ductería ø6" }\end{array}$} & \multicolumn{3}{|c|}{ Tubería PVC TDP 6" } & \multicolumn{3}{|c|}{$\begin{array}{c}\text { Instalación ductería PVC TDP 6" } \\
\text { para perforación }\end{array}$} \\
\hline & 1809 & COP 25 166,67 & COP 45526500,00 & 1809,0 & COP 7067,00 & COP 12784203,00 \\
\hline \multirow{2}{*}{$\begin{array}{l}\text { Perforación } \\
\text { ramming } 24 "\end{array}$} & \multicolumn{3}{|c|}{$\mathrm{N} / \mathrm{A}$} & \multicolumn{3}{|c|}{$\begin{array}{l}\text { Perforación ramming } 24 \text { " incluye } \\
\text { tubería en acero al carbón }\end{array}$} \\
\hline & & & COP - & 52,5 & COP 2179239,00 & COP 114410047,50 \\
\hline \multirow{2}{*}{$\begin{array}{l}\text { Perforación } \\
\text { ramming } 30 "\end{array}$} & \multicolumn{3}{|c|}{$\mathrm{N} / \mathrm{A}$} & \multicolumn{3}{|c|}{$\begin{array}{l}\text { Perforación ramming } 30 " \text { incluye } \\
\text { tubería en acero al carbón }\end{array}$} \\
\hline & & & COP - & 124,5 & COP 2974050,97 & COP 370269345,77 \\
\hline Total: & \multicolumn{3}{|r|}{ COP 45526500,00} & \multicolumn{3}{|r|}{ COP 497463596,27} \\
\hline
\end{tabular}

Fuente: elaboración propia

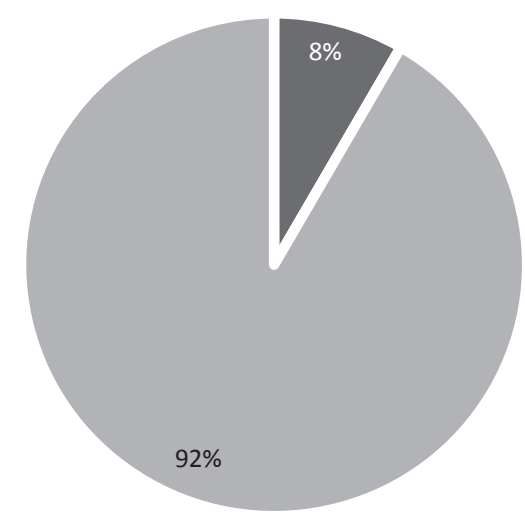

- Obra a cielo abierto Obra con sistema ramming

Figura 4. Comparativo de costos en instalación y suministros de tubería

Fuente: elaboración propia

Tabla 3. Comparativo costos en rellenos

\subsubsection{Costos directos en rellenos}

En el capítulo de rellenos se puede detectar la variación de cantidades especialmente en este ítem de excavaciones, puesto que en el sistema a cielo abierto se aumenta representativamente la cantidad de rellenos y el tipo de rellenos a utilizar, tal como se describe en la tabla 3.

De la tabla 3 y la figura 5 se evidenció que en el sistema tradicional a cielo abierto el costo se incrementa en un $44 \%$, en comparación con el sistema ramming, lo cual radica en la cantidad de rellenos que se duplica y del relleno en arena de peña requerido solo en el de a cielo abierto, de acuerdo con su proceso constructivo.

\begin{tabular}{|c|c|c|c|c|c|c|}
\hline & \multicolumn{3}{|c|}{ Obra a cielo abierto } & \multicolumn{3}{|c|}{ Obra con sistema ramming } \\
\hline Actividad & Cant. & Vlr. unitario & Actividad & Cant. & Vlr. unitario & Actividad \\
\hline \multirow{6}{*}{ Rellenos } & \multicolumn{3}{|c|}{ Relleno en sub base granular Clase в } & \multicolumn{3}{|c|}{ Relleno en subbase granular Clase в } \\
\hline & 51,7 & COP 125796,00 & COP 6508470,24 & 25,0 & COP 125796,00 & COP 3145906,37 \\
\hline & \multicolumn{3}{|c|}{ Relleno en arena de peña } & \multicolumn{3}{|c|}{ N/A } \\
\hline & 34,0 & COP 70778,00 & COP 2403942,04 & & & \\
\hline & \multicolumn{3}{|c|}{$\begin{array}{l}\text { Relleno en material seleccionado } \\
\text { proveniente de excavación }\left(\mathrm{m}^{3}\right)\end{array}$} & \multicolumn{3}{|c|}{$\begin{array}{l}\text { Relleno en material seleccionado } \\
\text { proveniente de excavación }\left(\mathrm{m}^{3}\right)\end{array}$} \\
\hline & 207,0 & COP 18773,00 & COP 3885131,86 & 100,0 & COP 18773,00 & COP 1877900,74 \\
\hline Total: & \multicolumn{3}{|r|}{ COP 12797544,14} & \multicolumn{3}{|r|}{ COP 5023807,10} \\
\hline
\end{tabular}

Fuente: elaboración propia 


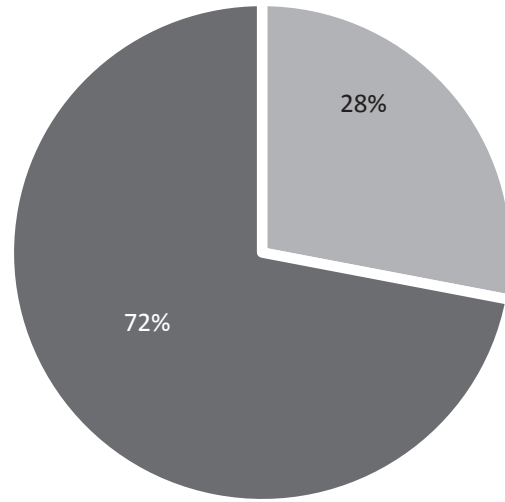

口 Obra a cielo abierto Obra con sistema ramming

Figura 5. Comparativo de costos en rellenos

Fuente: elaboración propia

\subsubsection{Costos directos en actividades de reparación de espacio público}

Una vez terminadas las actividades de obra civil se debe reparar el área intervenida en espacio público, para lo cual se deben realizar las actividades que se requieran. En la tabla 4 se describe la comparación.

Según la tabla 4 y la figura 6 , se evidenció que las actividades con mayor sobrecosto en el sistema a cielo abierto son la reparación de andén y de calzada; esta última no se requiere reparar en el sistema ramming, lo cual es uno de los beneficios, y no ocasiona ninguna afectación a la circulación vehicular de calzadas .

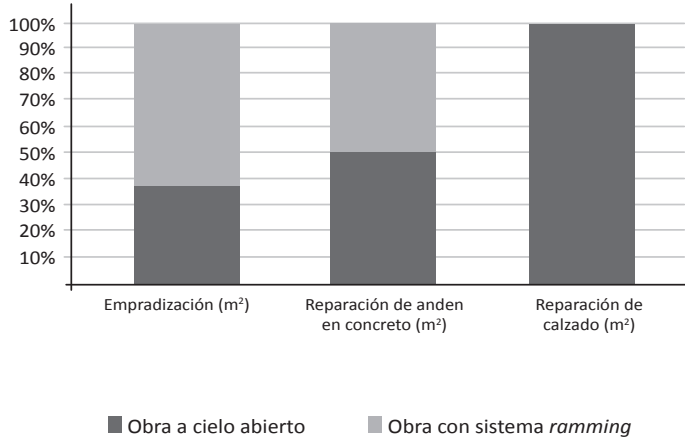

Figura 6. Comparativo costos actividades de reparación de espacio público

Fuente: elaboración propia

De acuerdo con el comparativo de cada una de las actividades anteriores se pudo recolectar la información y hallar un consolidado general, en el cual se comprobó que, en un análisis económico del costo directo de obra, ambos sistemas tuvieron variación de incrementos y declives de costo.

En el consolidado se pudo constatar el sobrecosto de las actividades más representativas del sistema ramming, en especial las perforaciones de 24 " y 30 " que equivalen a la diferencia de costo total directo entre las dos opciones de obra. La diferencia equivale a un porcentaje del $69 \%$ y el costo de estas actividades en el sistema ramming representa un $79 \%$.

Tabla 4. Comparativo de costos en actividades de reparación de espacio público

\begin{tabular}{|c|c|c|c|c|c|c|}
\hline & \multicolumn{3}{|c|}{ Obra a cielo abierto } & \multicolumn{3}{|c|}{ Obra con sistema ramming } \\
\hline Actividad & Cant. & Vlr. unitario & Actividad & Cant. & Vlr. unitario & Actividad \\
\hline \multirow{2}{*}{$\begin{array}{l}\text { Empradización } \\
\left(\mathrm{m}^{2}\right)\end{array}$} & \multicolumn{3}{|c|}{ Empradizarían (incluye $10 \mathrm{~cm}$ de tierra negra) } & \multicolumn{3}{|c|}{ Empradizarían (incluye $10 \mathrm{~cm}$ de tierra negra) } \\
\hline & 7,8 & COP 12 192,68 & COP 94810,28 & 12,0 & COP 12 192,68 & COP 146312,16 \\
\hline \multirow{4}{*}{$\begin{array}{l}\text { Reparación de an- } \\
\text { den en concreto } \\
\left(\mathrm{m}^{2}\right)\end{array}$} & \multicolumn{3}{|c|}{ Placa base concreto e $=0,10 \mathrm{~m} 2500$ psi } & \multicolumn{3}{|c|}{ Placa base concreto e $=0,10 \mathrm{~m} 2500$ psi } \\
\hline & 31,1 & COP 44300,45 & COP 1377921,20 & 48,0 & COP 44300,45 & COP 2126421,60 \\
\hline & \multicolumn{3}{|c|}{ Bordillo prefabricado de anden } & \multicolumn{3}{|c|}{$\mathrm{N} / \mathrm{A}$} \\
\hline & 21,6 & COP 42596,85 & COP 920091,96 & & $\mathrm{COP}-$ & $\$-$ \\
\hline \multirow{2}{*}{$\begin{array}{l}\text { Reparación de } \\
\text { calzada } \\
\left(\mathrm{m}^{2}\right)\end{array}$} & \multicolumn{3}{|c|}{$\begin{array}{l}\text { mezcla densa en caliente para reparación } \\
\text { calzada (incluye cemento asfaltico) }\end{array}$} & \multicolumn{3}{|c|}{$\mathrm{N} / \mathrm{A}$} \\
\hline & 141,6 & COP 160536,83 & COP 22732015,13 & & & $\mathrm{COP}-$ \\
\hline Total: & \multicolumn{3}{|r|}{ COP 25124838,56} & \multicolumn{3}{|r|}{ COP 2272733,76} \\
\hline
\end{tabular}

Fuente: elaboración propia 
Es importante señalar que sin esta actividad de perforación horizontal, en el comparativo con el sistema tradicional, el sistema ramming tendría hasta un $10 \%$ menos de costo. En los capítulos que es evidente el incremento de costo en la obra a cielo abierto se encuentran las actividades preliminares, los rellenos y la reparación de espacio público, actividades de mayor impacto ambiental y social para la ciudadanía.

\subsubsection{Estimación de los costos indirectos}

Dada la complejidad para estimar los costos sociales y ambientales en la zona de trabajo y en el momento que se realizó la investigación, se tomó como referencia estudios realizados en el mundo que sirviesen como base fundamental para obtener estos costos indirectos y, a su vez, el costo real del proyecto en estudio de zanja abierta.

Según el estudio que efectuó Apeldoorn sobre los costos indirectos que denominó "perjuicios colaterales" para los casos "Storm drain, Oackland, California", "Sewer 7 district Vienna, Austria" y "Sewer 19 district Vienna, Austria", en los que se tuvieron presentes aspectos sociales y ambientales, entre ellos los costos operativos de los vehículos, los costos debido a demoras de viaje, el costo de interrupción peatonal, el costo por contaminación de polvo y suciedad, el costo por pérdida de ingresos por parquímetros, el costo de la seguridad de los trabajadores y la disminución del valor de carretera [6, p. 12]. En esta parte de la investigación se utilizó como referencia la tabla resumen de Apeldoorn (según se aprecia en la tabla 5) con los datos de costos unitarios para cada categoría; la tabla muestra el mínimo y máximo valor por metro de tubería instalada y también el valor por día de trabajo.

Una vez analizada la información de la tabla 5 se procedió a convertir los valores a pesos colombianos por metro lineal de instalación y las cantidades de obra en metro lineal del caso de referencia, con el fin de obtener un rango de valores que oscilan entre un valor mínimo y un máximo, hasta alcanzar como resultado un promedio de costo de COP 435393 450,00.

\subsubsection{Consolidado de comparativo de costos directos e indirectos}

De acuerdo con el análisis tanto de costos directos de obra como de los costos indirectos que ocasionan perjuicios colaterales en el sistema tradicional a cielo abierto, se retomaron costos totales de cada sistema y se obtuvo el resultado que se presenta en la tabla 7.

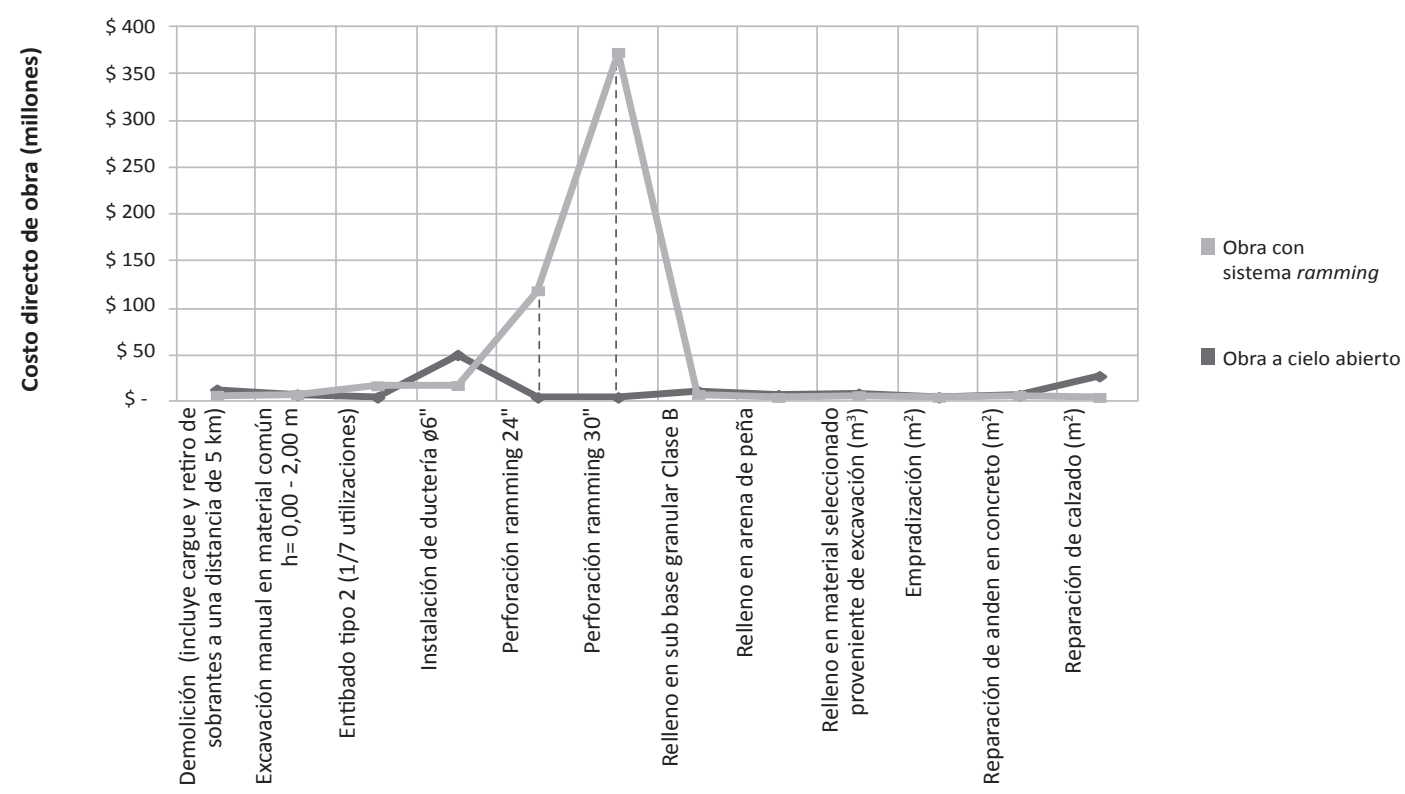

Figura 7. Comparativo consolidado de costos directos entre sistemas Fuente: elaboración propia 
El resultado recolectado en la tabla 7 demuestra un sobrecosto del $1,43 \%$ del sistema de perforación horizontal ramming, asumiendo un valor promedio con respecto al cálculo proyectado a los perjuicios colaterales de la obra a cielo abierto, el cual puede aumentar a un $71,40 \%$ si tomamos el valor máximo que pueden acarrear los costos indirectos en este tipo de obras.

Tabla 5. Valores máximos y mínimos de los costos sociales y ambientales por pie de tubería instalada y por día de trabajo

\begin{tabular}{|c|c|c|c|c|}
\hline Categoría de costo social & Minium $[\$ / \mathrm{m}]$ & Maximum $[\$ / \mathrm{m}]$ & Minium [\$/day] & Maximum $[\$ / \mathrm{m}]$ \\
\hline Costo de operación de vehículo & 9 & 271 & 26 & 1973 \\
\hline Costos de retraso de viaje & 13 & 940 & 41 & 6435 \\
\hline Polvo y tierra & 13 & 66 & 55 & 136 \\
\hline Ingresos de parquímetro & 26 & 39 & 83 & 217 \\
\hline $\begin{array}{l}\text { Disminución en el valor de la superficie } \\
\text { de la carretera }\end{array}$ & 66 & 144 & 227 & 318 \\
\hline
\end{tabular}

Fuente: [6]

Tabla 6. Cálculo de costos indirectos del proyecto en estudio de acuerdo con Apeldoorn

\begin{tabular}{|c|c|c|c|c|c|}
\hline \multirow[b]{2}{*}{ Costos indirectos } & \multirow[b]{2}{*}{ Cant. (ml) } & \multicolumn{2}{|c|}{ Mínimo } & \multicolumn{2}{|c|}{ Máximo } \\
\hline & & $\begin{array}{c}\text { Valor unitario } \\
(\text { tmr cop } 3100)\end{array}$ & $\begin{array}{l}\text { Valor mínimo } x \\
\text { ml de instalación }\end{array}$ & $\begin{array}{l}\text { Valor unitario } \\
(\text { tmr cop } 3100)\end{array}$ & $\begin{array}{l}\text { Valor máximo } \mathrm{x} \\
\text { ml de instalación }\end{array}$ \\
\hline $\begin{array}{l}\text { Operación } \\
\text { de vehículos }\end{array}$ & 177 & COP 27900,00 & COP 4938300,00 & COP 840 100,00 & COP 148697700,00 \\
\hline Demora de viajes & 177 & COP 40300,00 & COP 7133100,00 & COP 2914000,00 & COP 515778000,00 \\
\hline Polvo y suciedad & 177 & COP 40300,00 & COP 7133100,00 & COP 204 600,00 & COP 36214200,00 \\
\hline Ingresos parquímetro & 177 & COP 80600,00 & COP 14266200,00 & COP 120900,00 & COP 21399300,00 \\
\hline $\begin{array}{l}\text { Disminución del valor } \\
\text { de la superficie de la } \\
\text { calzada }\end{array}$ & 177 & COP 204 600,00 & COP 6214200,00 & COP 446400,00 & COP 79012800,00 \\
\hline $\begin{array}{l}\text { Valor total costos } \\
\text { indirectos: }\end{array}$ & \multicolumn{3}{|c|}{ COP 69684900,00} & \multicolumn{2}{|c|}{ СОР 801102000,00} \\
\hline Costo promedio: & & & & & COP 435393450,00 \\
\hline
\end{tabular}

Fuente: [6]

Tabla 7. Comparativo consolidado de costos directos e indirectos

\begin{tabular}{ccc}
\hline Actividades & Obra a cielo abierto & Obra con sistema ramming \\
\hline Total costo directo: & COP 94699223,87 & COP 522 623 712,73 \\
Total costo indirecto: & COP 435393450,00 & COP 0,00 \\
\hline Costo área total: & COP 530092673,87 & COP 522 623 712,73 \\
\hline
\end{tabular}




\subsection{Comparación desde el aspecto técnico}

Tabla 8. Comparativo de los aspectos técnicos en ambos sistemas constructivos

\begin{tabular}{|c|c|}
\hline Excavación a cielo abierto & Tecnología sin zanjas ramming \\
\hline $\begin{array}{l}\text { Se realiza la ruptura de vías. Por medio de excavaciones } \\
\text { (ocasionando su cierre), en los extremos de cada tramo se } \\
\text { realizan las cajas de inspección. }\end{array}$ & $\begin{array}{l}\text { Se realizan dos excavaciones, una caja de lance (en el inicio } \\
\text { del tramo) y una caja de recibo (al final de este); estas exca- } \\
\text { vaciones se ubican en zonas que no afecten las vías, como, } \\
\text { por ejemplo, andenes, zonas verdes, etc. }\end{array}$ \\
\hline $\begin{array}{l}\text { Se emplean equipos pesados (retroexcavadora, minicarga- } \\
\text { do) para la excavación y el relleno del tramo a intervenir. }\end{array}$ & $\begin{array}{l}\text { Se emplea el equipo de perforación acompañado del } \\
\text { compresor. }\end{array}$ \\
\hline $\begin{array}{l}\text { Una vez se tenga la excavación perfilada se extiende una } \\
\text { capa de arena en el fondo de la excavación, se instala la } \\
\text { primera sección de tubería PVC TDP, y se repite el procedi- } \\
\text { miento hasta terminar de instalar la tubería requerida (ya } \\
\text { sea de seis o de } 12 \text { tubos). }\end{array}$ & $\begin{array}{l}\text { Una vez se tenga la tubería en acero hincada se realiza la } \\
\text { limpieza y se procede a la instalación de la tubería PVC TDP, } \\
\text { no se requiere ningún material de atraque. }\end{array}$ \\
\hline $\begin{array}{l}\text { Sobre la última sección de tubería se extiende la última } \\
\text { capa de arena; una vez lista esta capa se extiende y compac- } \\
\text { ta una capa de subbase granular clase B. }\end{array}$ & $\begin{array}{l}\text { Una vez se tiene la tubería PVC TDP no es necesario exten- } \\
\text { der ningún material de relleno. }\end{array}$ \\
\hline $\begin{array}{l}\text { Posteriormente, se procede a realizar la reparación corres- } \\
\text { pondiente, es decir : vías (reparación de la calzada capa de } \\
\text { asfalto), andenes (reparación placas de concreto y adoquín) } \\
\text { y zona verde (empradización del sector). }\end{array}$ & $\begin{array}{l}\text { La reposición se realiza en andenes; en concreto o adoquín } \\
\text { y en zona verde (empradización). }\end{array}$ \\
\hline $\begin{array}{l}\text { La comisión de topografía hace el levantamiento del área } \\
\text { a intervenir para la realización de los diseños, inspecciona } \\
\text { redes existentes, vías alternas, etc., y por último hace el } \\
\text { levantamiento para los planos record. }\end{array}$ & $\begin{array}{l}\text { La comisión de topografía debe realizar chequeo constante } \\
\text { del alineamiento y nivel del tubo en acero, antes de iniciar } \\
\text { las actividades realiza la inspección de las redes existentes. }\end{array}$ \\
\hline $\begin{array}{l}\text { Referente al personal requerido: } \\
01 \text { Operador de la retroexcavadora. } \\
01 \text { Operador del minicargador. } \\
01 \text { Ayudante que inspecciona tuberías existentes para evitar } \\
\text { daños. } \\
01 \text { Oficial y } 01 \text { ayudante para la instalación de la tubería. } \\
01 \text { Ayudante que se encarga de la perfilada de la excava- } \\
\text { ción. } \\
02 \text { Ayudantes que encargan de cubrir la excavación. }\end{array}$ & $\begin{array}{l}\text { Referente al personal requerido: } \\
01 \text { Operador del ramming. } \\
02 \text { Trabajadores para limpieza del interior de la tubería. } \\
01 \text { Topógrafo y } 01 \text { cadenero. }\end{array}$ \\
\hline
\end{tabular}

Fuente: elaboración propia

\subsection{Comparativo consolidado de tiempos de ejecución}

A fin de lograr una comparación en duración de ejecución de obra entre el sistema con zanja y el sistema sin zanja por medio de la tecnología ramming se tomó la programación de obra ejecutada en el caso base "Expansión y mejoramiento del servicio de energía eléctrica”, en el municipio de Tocancipá, y se comparó de acuerdo con la duración requerida para las actividades que se hubiesen requerido en el sistema con zanja abierta.

De acuerdo con la comparación realizada en la figura 8 y teniendo en cuenta el proceso constructivo de cada uno de los sistemas se concluyó que el sistema ramming requiere la mitad de tiempo de ejecución con respecto al sistema de excavación a cielo abierto. En la comparativa de la programación se reflejó un valor de 56 días que se requiere adicionar para la ejecución de este proyecto con el sistema a cielo abierto.

\section{Discusión y conclusiones}

La construcción por medio del uso de tecnologías sin zanjas ramming para la instalación de redes subterráneas tuvo un costo en su proceso constructivo elevado con relación al sistema convencional de excavación a cielo abierto. Sin embargo, los costos 


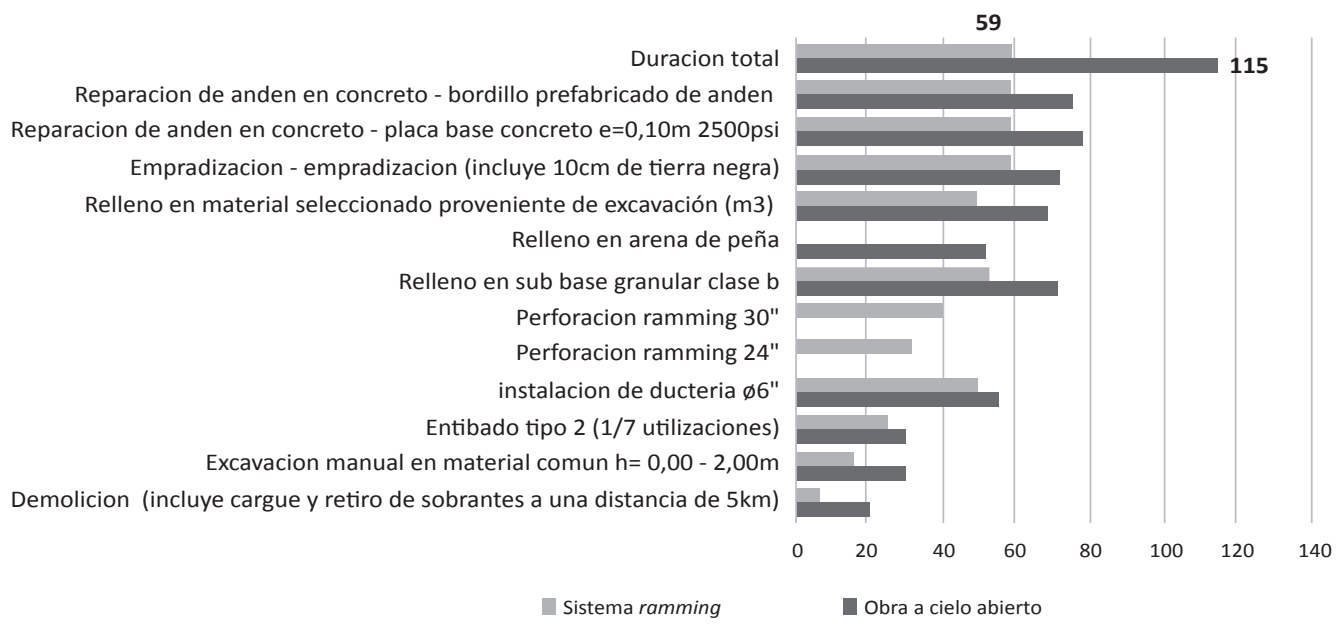

Figura 8. Comparativo de duración acumulada por actividad entre sistema ramming y tradicional a cielo abierto Fuente: elaboración propia

indirectos tanto ambientales como sociales son mínimos debido a que por este método se evitan afecciones que pueden producirse a las industrias, áreas comerciales, accesos a hospitales, salidas de cuerpos de emergencia, pistas aéreas y, en general, a cualquier infraestructura urbana. Este aspecto que es de gran consideración y valoración de cara al ordenamiento territorial.

En el consolidado del comparativo de los costos directos de obra se pudo observar el sobrecosto de las actividades más representativas del sistema ramming tales como las perforaciones de tuberías de diámetro de 24 " y 30 ", las cuales equivalen a un costo superior a la diferencia de costo total directo entre las dos opciones de obra. La diferencia equivale a un porcentaje del $69 \%$ y el costo de estas actividades en el sistema ramming representa un 79\%.

En los capítulos de "Rellenos" y "Reparación del espacio público" se comprobó el incremento de costo directo en la obra a cielo abierto en actividades tales como rellenos en subbase granular, y relleno en arena de cantera el incremento del costo es de un $40 \%$ con respecto al sistema ramming. Asimismo, en actividades como reparación de andén y calzada se pudo apreciar el sobrecosto puesto que la ejecución de esta última no es necesaria por el sistema ramming, lo que generó menor impacto ambiental y social para la ciudadanía.

Las actividades con mayor influencia de costo directo en el sistema tradicional a cielo abierto fueron la instalación de ductería que obtiene un $48 \%$, y la reparación de calzada con un $24 \%$, lo cual nos aclara cuáles ítems son los más costosos y requieren más tiempo en su ejecución.

En el caso del sistema de perforación horizontal ramming se observó un $83 \%$ de sobrecosto directo que genera en el comparativo consolidado contra la obra a cielo abierto.

Al consolidar un comparativo de costos directos e indirectos se demostró un sobrecosto del 1,43\% en el sistema de perforación horizontal ramming, asumiendo un valor promedio con respecto al cálculo proyectado a los perjuicios colaterales de la obra a cielo abierto, el cual puede aumentar a un $71,40 \%$ si tomamos el valor máximo que pueden acarrear los costos indirectos en este tipo de obras.

Con respecto a la revisión literaria hecha para la presente investigación se puede evidenciar que se han aportado datos que reafirman la necesidad de seguir apostando por nuevas tecnologías de construcción que ayuden a mitigar problemas sociales y ambientales que se generan de los métodos de construcción tradicionales. Esta investigación se ha aplicado en una zona industrial y los resultados han reflejado la importancia que tiene desarrollar nuevos métodos de construcción; no obstante, sería conveniente continuar la investigación y aplicar esta metodología a zonas densamente pobladas en las que los costos sociales y ambientales tengan un mayor rol dentro de la estimación de los costos totales. También se podría pensar en hacer una comparación técnica y económica con otros métodos de 
construcción sin zanjas que se puedan desarrollar en el país, como, por ejemplo, pipe jacking, horizontal directional drilling (HDD), horizontal auger boring (HAB), o microtunneling, entre otros.

\section{Referencias}

[1] J. Alarcón-Rocha y J. Pacheco-Calvo, “Comparación tecnológica y costos del método de instalación de tuberías sin zanja (trenchless) más eficiente para los suelos encontrados en un proyecto de Bogotá," trabajo de pregrado, Pontificia Universidad Javeriana, Bogotá, Colombia, 2014, 173 pp. [En línea]. Disponible en: http://hdl.handle.net/10554/16783

[2] J. C. Gutiérrez, "Method selection for trenchless technology (тT) in South America," tesis de maestría, Lousiana Tech University, Lousiana, EE. UU., 2006, 175 pp. [En línea]. Disponible en: http://www.grupo-epm.com/Portals/1/biblioteca_epm_virtual/tesis/Methodselectionfortrenchlesstechnology.pdf

[3] J. Pinzón-Abaunza. Evaluación y perspectivas de la utilización de tecnologías sin zanja en redes de alcantarillado de Bogotá. Bogotá, Colombia: Pontificia Universidad Javeriana, 2011, 159 pp. [En línea]. Disponible en: http://hdl.handle.net/10554/7549

[4] R. L. Sterling, "Guidlines for pipe ramming”, U. S. Army Corps of Engineers, Vicksburg, Technical Report number: Trenchless Technology Center Technical Report \#2001.04, 29 pp., 2001. [En línea]. Disponible en: http://citeseerx.ist.psu.edu/viewdoc/ download?doi=10.1.1.509.6025\&rep=rep1\&type $=\mathrm{pdf}$

[5] A. W. Stuedlein, "Analysis and desing of pipe ramming installations", Oregon Deparment of Transportation and Federal Highway Administration, Salem, OR, EEUU, Technical Report number: SPR 710, 236 pp., 2013. [En línea]. Disponible en: https://digital. osl.state.or.us/islandora/object/osl:40172

[6] S. Apeldoorn, "Comparing the cost-trenchless versus traditional methods", en International Society for Trenchless Technology Conferencie, Australasian Society for Trenchless Technology, Sidney, 2010, 8 pp. [En línea]. Disponible en: https://www.waternz. org.nz/Attachment?Action=Download\&Attachment_id=1344p

[7] J. Schill, "Pipe ramming proves golden for Colorado HDD project", Underground Construction, vol. 71, no. 11, pp. 28-29, nov. 2016. [En línea]. Disponible en: http://connection.ebscohost.com/c/articles/120244 677/pipe-ramming-proves-golden-colorado-hdd projec

[8] A. M. Stocking, "Highway monitoring for complex pipe jacking projects”, Point of Beginning, vol.
40, no. 5, pp. 10-12, feb. 2015. [En línea]. Disponible en: http://digital.bnpmedia.com/allarticle/12060/243551/243551/allarticle.html

[9] Y.Hashash y J. Javier, "Evaluation of horizontal directional drilling (HDD)”, Illinois Center for Transportation, Illinois, EE. UU., Technical Report number: ICT-R27-SP16, 51 pp., 2011. [En línea]. Disponible en: https://experts.illinois.edu/en/publications/evaluation-of-horizontal-directional-drilling-hdd

[10] A. Atalah y S. Bromn, "Development of standard specification for horizontal directional drilling", OhioDeparmentofTransportation, Columbus, Ohio, EE. UU., Technical Report number: FHWA/OH-2013/2, 52 pp., 2013. [En línea]. Disponible en: http://www. dot.state.oh.us/Divisions/Planning/SPR/Research/ reportsandplans/Reports/2013/Construction/13 4628_FR.pdf

[11] D. M. Abraham, H. Baik, y S. B. Gokhale, "Development of decision support system for selection of trenchless technologies to minimize impact of utility construction on roadways", Indiana Department of Transportation and Purdue University, Springfield, Project number: C-36-67HнH, 171 pp., 2007, doi: https://doi.org/10.5703/1288284313183

[12] N. Howell, “The pipe ramming technique”, en Sewers, pp. 254-271, 2004. doi: https://doi.org/10.1016/ B978-075065083-0/50014-1

[13] O. Collins, "Pneumatic pipe ramming solves emergency situation for rail corridor", en International Conference on Pipeline Engineering and Construction, Boston, Massachusetts, EE. UU., jul. 8-11, 2007. doi: https://doi.org/10.1061/40934(252)104

[14] A. W. Stuedlein y T. Meskele, "Preliminary design and engineering of pipe ramming installations", Journal of Pipeline Systems Engineering \& Practice, vol. 3, no. 4, pp. 125-134, nov. 2012. doi: https://doi. org/10.1061/(ASCE)PS.1949-1204.0000107

[15] G. W. E. Milligan y C. D. F. Rogers, "Trenchless technology", en Geotechnical and geoenvironmental engineering handbook, Norwell, MA, EE. UU.: Kluwer Academic Publishing, pp. 569-592, 2001. doi: https://doi.org/10.1007/978-1-4615-1729-0_19

[16] S. R. Kramer, W. J. McDonald y J. C. Thomson. An introduction to trenchless technology. Nueva York: Van Nostrand Renhod Publisher, pp. 122-132, 1992. doi: 10.1007/978-1-4615-3058-9

[17] D. Stein. Trenchless technology for installation of cables and pipelines. $1^{\text {a }}$ ed., Stein and Partners, 2005, $866 \mathrm{pp}$.

[18] S. Ariaratnam, W. Chan y D. Choi, "Utilization of trnchless construction methods in mainland China to sustain urban infraestructure", Practice Periodical on Structural Design and Construction, vol. 3, 
no. 3,pp. 134-141,2006. doi:https://doi.org/10.1061/ (ASCE)1084-0680(2006)11:3(134)

[19] T. Meskeley A. Stuedlein, "Analysis of a 610-mm-diameter pipe installed using pipe ramming", Journal of Performance of Constructed Facilities, vol. viII, n. ${ }^{\circ}$ 4, ag. 2014. doi: https://doi.org/10.1061/(ASCE) CF.1943-5509.0000463

[20] M. Najafi. Pipe ramming projects. EE. UU.: American Society of Civil Engineering, 2008. doi: https://doi. org/10.1061/9780784409794

[21] J. Matthews, E. Allouche y R. Sterling, "Social cost impact assessment of pipeline infrastructure projects", Environmental Impact Assessment Review, vol. 50, pp. 196-202, 2015. doi: http://dx.doi.org/ 10.1016/j.eiar.2014.10.001

[22] J. Yeun J. y S. Sunil, "Evaluation of trenchless technology methods for municipal infrastructure system, Journal of Infrastructure Systems, vol. 13, no. 2, pp. 144-156, jun. 2007. doi: https://doi.org/10.1061/ (ASCE)1076-0342(2007)13:2(144)

[23] V.Gerasimova, "Underground engineering and trenchless technologies at the defense of environment", Procedia Engineering, no. 165, pp. 1395-1401, 2016. doi: https://doi.org/10.1016/j.proeng.2016.11.870

[24] S. T. Ariaratnam, "Sustainable development through innovative underground infrastructure construction practices," en International Conference on Structural Engineering Construction and Management, Sri Lanka, 2011, pp. 1-11. [En línea]. Disponible en: http://dl.lib.mrt.ac.lk/handle/123/9383

[25] North American Society for Trenchless Technology, "Nasttghgcalculator", Nasttghgcalculator.com. [En línea]. Disponible en: www.nastt.org
[26] M. Myers, T. Stickrod, D. Abraham y T. Iseley, "Microtunneling technology for conduit construction", Practice Periodical on Structural Design and Construction, vol. 4, no. 2, pp. 56-63, 1999. doi: https:// doi.org/10.1061/(ASCE)1084-0680(1999)4:2(56)

[27] C. Pupo-González, "Metodología para la selección de obras de ingeniería para la rehabilitación de redes troncales de alcantarillado", tesis de maestría, Escuela Colombiana de Ingeniería, Bogotá, 2014, 152 pp. [En línea]. Disponible en: https://repositorio.escuelaing.edu.co/bitstream/001/125/1/Tesis\%20Documento\%20Final.pdf

[28] F. Mínguez-Santiago, "Métodos de excavación sin zanjas”, tesis doctoral, Universidad Politécnica de Madrid, Madrid, España, 2015, 82 pp. [En línea]. Disponible en: http://oa.upm.es/37225/1/Tesis_ master_Felicidad_Minguez_Santiago.pdf

[29] P. Marín-Ibarra, "Estudio de la factibilidad técnica y económica del uso del nuevo sistema de instalación y/o reemplazo de tuberías, sin zanjas, para conducciones de agua potable", tesis de maestría, Universidad Industrial de Santander, Bucaramanga, 2004, 149 pp. [En línea]. Disponible en: https://documentslide.org/embed/113792-pdf

[30] Pinter \& Associates Ltd., “Trenchless technologies and work practices review for Saskatchewan municipalitie", Communities of tomorrow, Eslovaquia, 2013. [En línea]. Disponible en: https://suma.org/ img/uploads/documents/ communities_of_tomorrow/Trenchless\%20Technologies.pdf

[31] Asociación Ibérica de Tecnología Sin Zanja. Manual de tecnologías sin zanja. $2^{\text {a }}$ ed., Madrid, España: Asociación Ibérica de Tecnología Sin Zanja, 2016, 333 pp. 